\title{
The relative role of EUV radiation and X-rays in the heating of hydrogen-rich exoplanet atmospheres
}

\author{
C. Cecchi-Pestellini ${ }^{1}$, A. Ciaravella ${ }^{2}$, G. Micela ${ }^{2}$, and T. Penz ${ }^{2}$ \\ 1 INAF - Osservatorio Astronomico di Cagliari, Strada 54, Loc. Poggio dei Pini, 09012 Capoterra (CA), Italy \\ e-mail: ccp@ca.astro.it \\ 2 INAF - Osservatorio Astronomico di Palermo, Piazza Parlamento 1, 90134 Palermo, Italy \\ e-mail: [ciarave;giusi; tpenz]@astropa.unipa.it
}

Received 11 April 2008 / Accepted 5 January 2009

\section{ABSTRACT}

\begin{abstract}
Aims. We study the relative role of EUV and X-ray radiation in the heating of hydrogen-rich planet atmospheres with different composition and electron content.

Methods. An accurate photo-ionization model has been used to follow the primary photo-electron energy deposition throughout the atmosphere.

Results. Heating rates and efficiencies have been computed, together with column density cut-offs at which photons of given energies stop their heating production inside the atmosphere. Assuming $100 \mathrm{eV}$ as the energy borderline between the extreme ultraviolet spectral range and X-rays we find that when the absorbing hydrogen column density is higher than $10^{20} \mathrm{~cm}^{-2}$ only X-rays can heat the gas. Extreme ultraviolet photons heat the upper atmospheric layers.

Conclusions. Using emission spectra from a sample of solar-type stars of different ages representative of the Sun's main sequence lifetime, we have derived the corresponding heating rates. We find that the existence of an energetic cross-over in atmospheric heating is present for all stars in the sample.
\end{abstract}

Key words. stars: planetary systems - ultraviolet: stars - X-rays: stars - radiative transfer

\section{Introduction}

Recent observations of the planet HD 209458b indicate that it is surrounded by an expanded atmosphere of atomic hydrogen, escaping hydrodynamically (Vidal-Madjar et al. 2003; Ballester et al. 2007) at a rate of $10^{10} \mathrm{~g} / \mathrm{s}$. Following the observation of Vidal-Madjar et al. (2003), several theoretical studies on expanded atmospheres around close-in gas giant planets were put forward. Lammer et al. (2003) and Baraffe et al. (2004, 2005) predicted loss rates as high as $10^{12} \mathrm{~g} / \mathrm{s}$ using a scaling law based on an approximate solution of the heat balance equation in the upper atmosphere of planets. Hydrodynamic models gave lower loss rates, of a few times $10^{10} \mathrm{~g} / \mathrm{s}$ (Yelle 2004; Tian et al. 2005; Yelle 2006; García Muñoz 2007).

However, recent studies (Hubbard et al. 2007) showed that the observed planetary mass distribution is not in agreement with the high mass loss rates predicted by Lammer et al. (2003) and Baraffe et al. (2004, 2005). A re-analysis of the Vidal-Madjar et al. (2003) HST data performed by Ben-Jaffel (2007), although confirming the detection of an expanded atmosphere, found no indications for mass loss rates of the order of $10^{10} \mathrm{~g} / \mathrm{s}$ (see, however, Vidal-Madjar et al. 2008; and Ehrenreich et al. 2008 , for a different interpretation of the data). A very recent study by Holmström et al. (2008) presents evidence that the atomic hydrogen absorption in the stellar Lyman- $\alpha$ by the planet HD 209458 b detected with HST is affected by energetic neutral atoms originating from the stellar wind interaction with the expanded atmosphere. Since a range of exospheric conditions and atmospheric loss rates are consistent with the observations,
Holmström et al. (2008) concluded that we have little information on the main exospheric component. Berdyugina et al. (2008) used polarized scattered light to gain information about the atmosphere of HD 189733b. They found an extended atmosphere, but to fill the Roche lobe, which would be required to get high loss rates, a very low albedo, $\sim 0.15$, is needed.

Cecchi-Pestellini et al. (2006) showed that X-rays give an important contribution to the heating of hydrogen-rich planetary atmospheres. Using just the X-ray heating channel, Penz et al. (2008) and Penz \& Micela (2008) investigated planetary mass loss from close-in, gas giant planets orbiting $\mathrm{dG}$ and $\mathrm{dM}$ stars, and showed that X-ray irradiation may affect the final planetary mass.

Indeed, from the study of stellar proxies for the Sun it appears that young solar-type stars emit X-rays at a level three to four orders of magnitude higher than the present-day Sun, both during the pre-main sequence phase when the emission is dominated by intense daily or weekly flares (Feigelson et al. 2003; Favata et al. 2005), and during the first phases of the main-sequence (Micela 2002). Such a copious emission must have affected the circumstellar disk and the process of planetary formation during the pre-main sequence phase, and subsequently, when the star reaches the main sequence, the evolution of planetary atmospheres. To estimate the effect of XUV emission (roughly the spectral range between 0.1 and $90 \mathrm{~nm}$ ) from solar-type stars of different ages we exploit the XUV emission of six stars from the Sun in Time program (Guinan \& Ribas 2002), whose fluxes are assumed to describe the evolution of the Sun's XUV emission (Ribas et al. 2005). From the Ribas et al. (2005) 
data, it appears that for a $100 \mathrm{Myr}$ star the ratio between the integrated fluxes in the extreme ultraviolet (EUV; 10-90 nm) and $\mathrm{X}$-rays $(0.1-10 \mathrm{~nm})$ is lower than unity, and it remains within a factor of two for stars as old as $1 \mathrm{Gyr}$ (the solar ratio is about 4). Moreover the star luminosity seems to be characterized by evolutionary time-scales that are different in different spectral bands. The X-ray luminosity evolution of $\mathrm{dM}$ stars is slower than that of dG stars, and may present frequent flaring activity. However, even if the ratio between the X-ray luminosity and the bolometric luminosity is typically larger in $\mathrm{dM}$ stars than in solar-type stars, the absolute value of the X-ray luminosity is lower at each age (see Figs. 2 and 3 of Penz \& Micela 2008). Therefore we expect that the effects of EUV stellar emission are less relevant at the same distance from the host stars. We note however that the habitable zone of $\mathrm{dM}$ stars is closer by a factor 10-100 to the star, depending on the star, and therefore the effects on planets in the habitable zone may be much more relevant.

Most of estimates of the extrasolar giant planet escape rates are based on a total conversion of the absorbed stellar XUV energy into powering escape. In the light of the above discussion, it is important to assign the fraction of stellar XUV radiation that goes into the heating of upper atmospheres of extrasolar giant planets, and to evaluate how deep in an atmosphere stellar photons, e.g. in the EUV spectral range, keep releasing part of their energy as heat. This task requires an accurate description of radiative transfer and photo-electron energy deposition (Cecchi-Pestellini et al. 2006).

The model is briefly described in Sect. 2, while results are given in Sect. 3. Discussion and conclusions are in Sect. 4.

\section{Model description}

The flux of stellar XUV photons incident upon a planetary atmosphere of solar-like composition photo-ionizes the gas producing a flux of high energy photo-electrons, which deposit their energy into the gas. In a partially neutral medium, electrons ionize, excite, and dissociate atomic and molecular species, as well as heat the gas through Coulomb collisions. In determining these energy deposition events, we must account for all the possible degradation histories of the energetic electrons. The derived yields allow the determination of the ionization, dissociation, excitation, and heating rates (Dalgarno et al. 1999).

When the stopping medium is only partially neutral, electron-electron interactions contribute to the electron energy degradation, and a significant portion of the fast electron energy is deposited into the stopping medium as heat. As the fractional ionization rises, more and more of the electron energy heats the gas, and the excitation and ionization yields decrease.

The electron energy degradation is characterized by the mean energy per ion pair, which is the initial energy of a photoelectron divided by the number of secondary ionizations produced as the fast primary electron comes to rest. In order to compute the loss process numerically, we must divide the primary electron energy in a series of bins and define the probabilities, computed by the cross-sections, that any bin is emptied by elastic and inelastic collisions. We use the method presented by Dalgarno et al. (1999, and references therein) in which each successive bin is emptied in turn until the energy falls below the threshold energy of the least energetic inelastic process. The residual energy is taken up as heat.

Since the gas photo-electric cross-section scales roughly as the inverse third power of the energy, the stellar spectrum becomes progressively weaker and harder as the depth inside the atmosphere increases. The local residual stellar spectrum is computed incorporating in the radiative transfer equation an accurate description of the relevant photo-electric processes: ionization of atomic hydrogen (Sobel'man 1991), helium (Yan et al. 1998), ionized helium (Osterbrock 1989), molecular hydrogen (Yan et al. 1998)), and Compton ionization, that, at energies $\gtrsim 10 \mathrm{keV}$, is more efficient than photo-ionization of $\mathrm{H}$ and $\mathrm{He}$ (Yan et al. 1998). Furthermore, we adopt relativistic photoionization cross-sections of individual heavy elements and their ions given in Verner et al. (1993). A detailed description of the model is found in Cecchi-Pestellini et al. (2006).

In radiative transfer calculations the relevant quantity is the column density of the absorbing material. We shall evaluate a location within the atmosphere in terms of the neutral hydrogen column density $N_{\mathrm{H}}$, measured starting from the top of the atmosphere. The photo-ionization heating rate at $N_{\mathrm{H}}$ is

$Q_{\mathrm{XUV}}\left(N_{\mathrm{H}}\right)=\sum_{k} \int\left[\frac{\mathrm{d} \mathcal{N}_{1}\left(E, N_{\mathrm{H}}\right)}{\mathrm{d} E} \times \mathcal{E}_{Q}\left(E-I_{k}\right)\right]_{k} \mathrm{~d} E$,

where $I_{k}$ is the ionization potential and $k$ runs over all ionization channels, $\mathcal{E}_{Q}$ is the fraction of primary photo-electron energy going into gas heating computed taking into account all secondary processes, and

$$
\frac{\mathrm{d} \mathcal{N}_{1}(E)}{\mathrm{d} E}=S_{\mathrm{XUV}}\left(E, N_{\mathrm{H}}\right) \times\left[\sigma_{\mathrm{pa}}(E)+\sigma_{\mathrm{C}}(E)\right]
$$

is the primary electron differential spectrum. In Eq. (2) $S_{\mathrm{XUV}}\left(E, N_{\mathrm{H}}\right)$ is the local radiation flux, $\sigma_{\mathrm{pa}}=\sum_{i} f_{i} \sigma_{i}$ is the effective photo-absorption cross-section of a gas mixture in which the $i$-th species has a concentration $f_{i}$ and an individual photo-absorption cross-section $\sigma_{i}$, while $\sigma_{\mathrm{C}}$ is the total Compton photo-ionization cross-section.

The dependence of the heating rate $Q_{\mathrm{XUV}}$ on the energy of the incoming stellar photon is studied using a monochromatic stellar photon flux $S_{\mathrm{XUV}}^{\star}=S_{0} \delta\left(E-E_{0}\right)$. Equation (1) becomes

$$
\begin{aligned}
Q_{\mathrm{XUV}}\left(N_{\mathrm{H}}\right)= & S_{0} \mathcal{E}_{Q}\left(E_{0}-\langle I\rangle\right) \\
& \times\left[\sigma_{\mathrm{pa}}\left(E_{0}\right)+\sigma_{\mathrm{C}}\left(E_{0}\right)\right] \exp \left[-\tau\left(N_{\mathrm{H}}, E_{0}\right)\right]
\end{aligned}
$$

where $\tau\left(N_{\mathrm{H}}\right)=\left(\sigma_{\mathrm{pa}}+\sigma_{\mathrm{C}}\right) \times N_{\mathrm{H}}$ is the optical depth, and $\langle I\rangle$ the average ionization potential of the atmospheric gas mixture.

Finally, the heating yield, i.e. the fraction of incident stellar radiation converted to heat at $N_{\mathrm{H}}$, is obtained integrating Eq. (3) along the absorption path

$$
Y_{Q}=\left(\int S_{\mathrm{XUV}}^{\star}(E) \mathrm{d} E\right)^{-1} \times \int_{0}^{N_{\mathrm{H}}} Q_{\mathrm{XUV}}\left(N_{\mathrm{H}}^{\prime}\right) \mathrm{d} N_{\mathrm{H}}^{\prime}
$$

\section{Results}

We consider an atmosphere of solar composition (Anders \& Grevesse 1989) with all elements in atomic form. We choose to parametrize the electron content in order to highlight the effect of the electron concentration on the heating rate. The heating rates due to an incident unit flux $S_{0}=1$ at various locations inside an atmosphere with a fractional electron concentration $x_{\mathrm{e}}=0.1$ are shown in Fig. 1. The heating rate in Fig. 1 does not change significantly if the hydrogen is in molecular form, while it is strongly dependent in absolute value on the electronic content (Dalgarno et al. 1999; Cecchi-Pestellini et al. 2006), decreasing by about a factor of four in an almost neutral gas $\left(x_{\mathrm{e}} \lesssim 10^{-6}\right)$.

An important parameter is the metallicity of the atmosphere. Figure 2 shows the variations in the heating rate profiles as functions of the atmospheres metallicity: metal-rich (3 times larger 


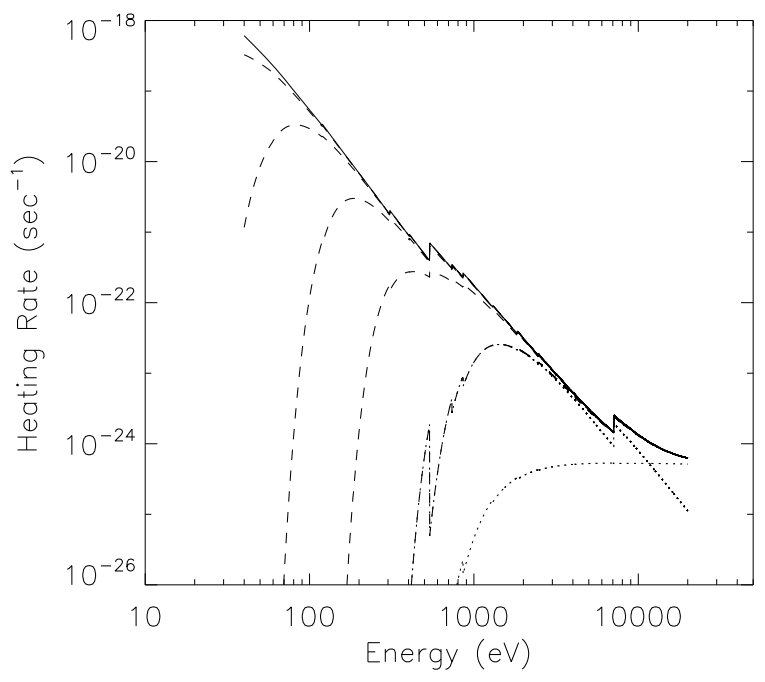

Fig. 1. Heating rate $\left(\mathrm{s}^{-1}\right)$, Eq. (3), for a monochromatic unit flux $\left(S_{0}=\right.$ $1)$ as a function of the photon energy in $\mathrm{eV}(1 \mathrm{keV}=1.24 \mathrm{~nm})$. The hydrogen is in atomic form and the electron fraction is $x_{\mathrm{e}}=0.1$. The heating rate is computed at the top of the atmosphere (solid line), and inside (dashed lines) at locations labelled with corresponding column densities $\log _{10}\left(N_{\mathrm{H}} / \mathrm{cm}^{-2}\right)=18,19,20,21,22$ (top to bottom). Dotted lines represent the contributions to the heating rate due to photo-absorption and Compton ionization channels when the hydrogen column density is $N_{\mathrm{H}}=10^{22} \mathrm{~cm}^{-2}$.

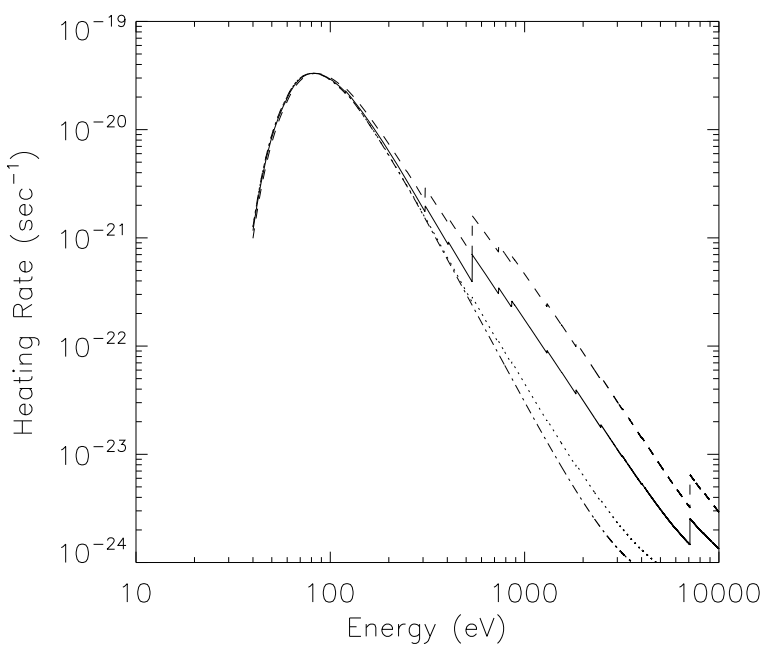

Fig. 2. Heating rate $\left(\mathrm{s}^{-1}\right)$, Eq. (3), for a monochromatic unit flux $\left(S_{0}=\right.$ 1 ) as a function of the photon energy $(\mathrm{eV})$ at $N_{\mathrm{H}}=10^{19} \mathrm{~cm}^{-2}$ for atmospheres of metallicities: 0.1 (dotted line), 1 (solid line) and 3 (dashed line) times the standard solar metallicity (Anders \& Grevesse 1989). Dot-dashed profile represents the heating rate computed for a pure $\mathrm{H}-\mathrm{He}$ atmosphere. The hydrogen is in atomic form and the electron fraction is $x_{\mathrm{e}}=0.1$.

than the solar values), solar and metal-poor (ten times less abundant than the solar values). In Fig. 2 we show an additional profile pertaining to a model atmosphere containing no heavy elements (just $\mathrm{H}$ and $\mathrm{He}$ ). At $1 \mathrm{keV}$, where the gas photo-absorption cross-section is dominated by ionization of heavy elements, the heating rate of a metal-rich atmosphere is about one order of magnitude higher than in the case of a metal-poor gas.

Results shown in Fig. 1 emphasize the existence of an energetic cross-over in the gas located approximately at $N_{\mathrm{H}}=$ $1-10 \times 10^{19} \mathrm{~cm}^{-2}$. For lower column densities gas heating is driven by EUV radiation, while X-rays dominate at deeper
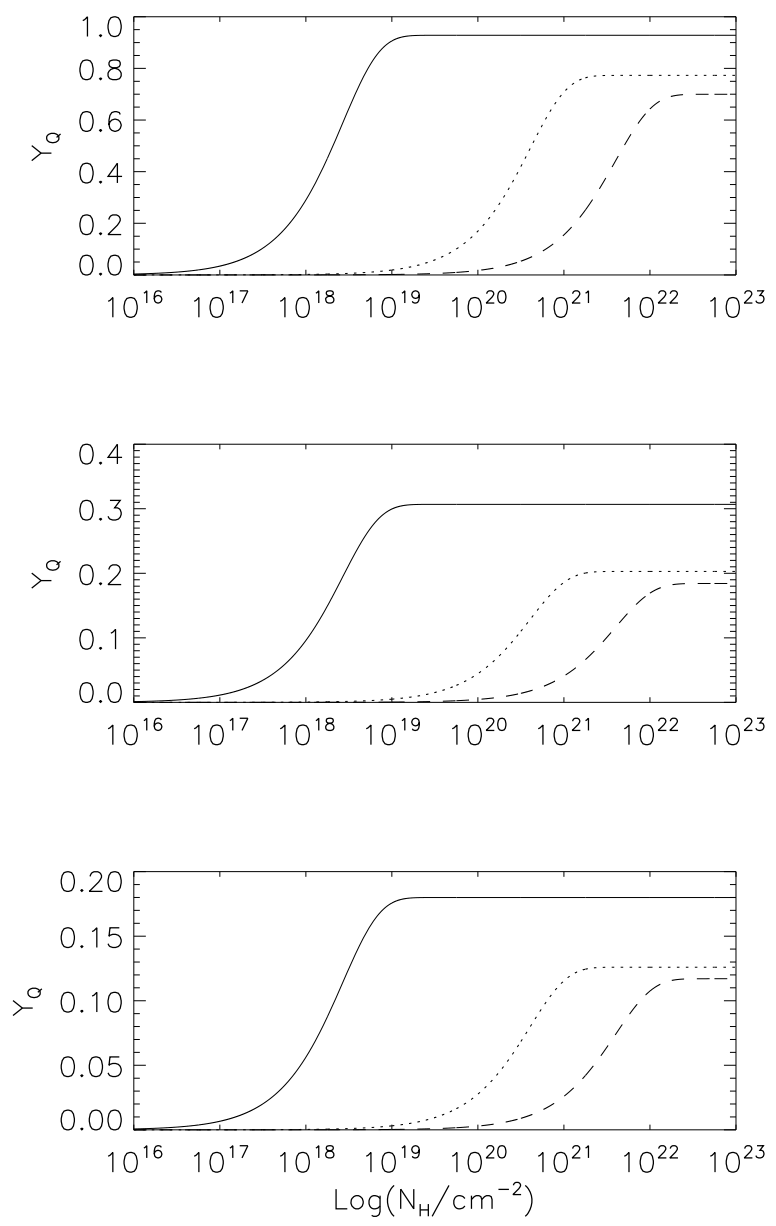

Fig. 3. Heating efficiency, Eq. (4), for a monochromatic unit flux as a function of the hydrogen column density of absorbing material $\left(\mathrm{cm}^{-2}\right)$ for three photon energies $E=50 \mathrm{eV}$ (solid line), $300 \mathrm{eV}$ (dotted line), and $1 \mathrm{keV}$ (dashed line). The gas has standard solar metallicity. The hydrogen is in atomic form and the electron fraction is $x_{\mathrm{e}}=0.1$ (top panel), $10^{-3}$ (middle panel), and $10^{-6}$ (bottom panel).

locations in the atmosphere. This is not surprising since high energy photons are preferentially absorbed by heavy elements (cf. Fig. 2), whose low abundances in solar-like mixtures produces a corresponding small optical depth, while in the EUV spectral range the main absorbers are hydrogen and helium.

The heating yield, Eq. (4), provides the screening length for photons of different energies. In Fig. 3 we plot $Y_{Q}$ for three values of the representative electronic fractions, $x_{\mathrm{e}}=$ $0.1,10^{-3}$, and $10^{-6}$, and for incoming stellar photons of energies 50,300 and $1000 \mathrm{eV}$ respectively as a function of the hydrogen column density. It is evident that the higher the stellar photon energy the deeper is the layer in the atmosphere in which the photon flux releases its energy. Of course, we do not have an $100 \%$ heating yield, because part of the photon energy is deposited in ionization and excitation of the atmospheric gas. Inelastic loss channels are more effective in a gas with low electronic content. As a consequence, the fraction of energy released in the heating of the gas is larger for higher electronic fractions. At sufficiently high hydrogen column density, $N_{\mathrm{H}}^{\mathrm{s}}$, the fraction of the incident radiation, $S_{\mathrm{XUV}}^{\star}$, that is converted to heat saturates at the mean heating efficiency

$Y_{Q} \sim\left\langle\mathcal{E}_{Q}\right\rangle=\frac{\int\left[S_{\mathrm{XUV}}\left(E, N_{\mathrm{H}}>N_{\mathrm{H}}^{\mathrm{s}}\right) \times \sum_{k} \mathcal{E}_{Q}\left(E-I_{k}\right)\right] \mathrm{d} E}{\int S_{\mathrm{XUV}}^{\star}(E) \mathrm{d} E}$. 


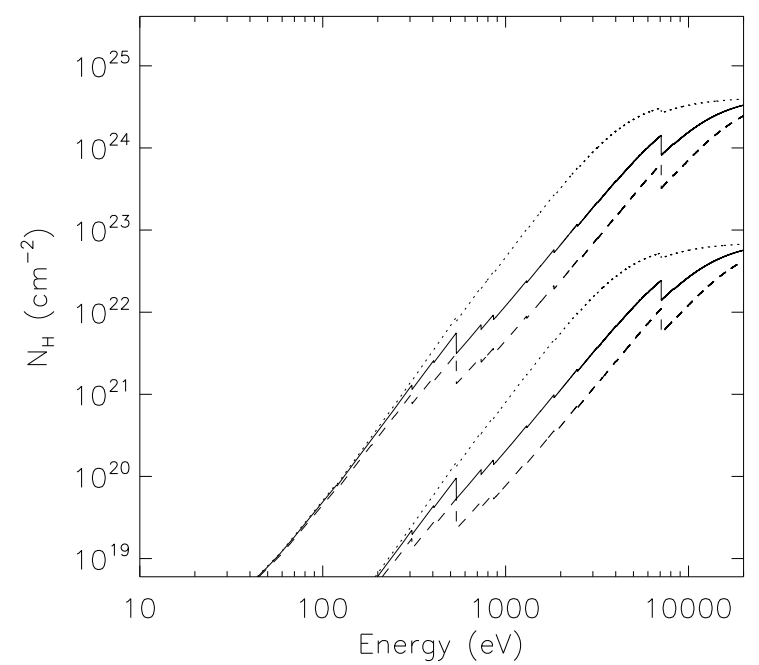

Fig. 4. Column density cut-offs (upper curves) as functions of the incident photon energy. The lower set of curves represents the column densities at which photons deposit $5 \%$ of their energy as heat. Dotted, solid and dashed lines correspond to atmosphere metallicities of 0.1 , 1 and 3 times the standard solar values (Anders \& Grevesse 1989). Atmospheric conditions are the same as in Fig. 3.

In the case of a monochromatic unit flux, $\left\langle\mathcal{E}_{Q}\right\rangle$ is the heating efficiency pertaining to the generated primary photo-electron of energy $E-\langle I\rangle$.

The column density cut-off $N_{\mathrm{H}}^{\mathrm{s}}$ at which a photon of energy $E$ stops its heating production inside the atmosphere is shown in Fig. 4. The upper layers of an atmosphere, $N_{\mathrm{H}} \lesssim 10^{20} \mathrm{~cm}^{-2}$, are almost transparent to photons of energies in the keV range. In Fig. 4 we also show the column density at which a photon deposits $5 \%$ of its energy as heat. The region between the two curves indicates the column density range where a photon of a given energy heats the material. For thin atmospheres $\left(N_{\mathrm{H}} \lesssim 10^{19} \mathrm{~cm}^{-2}\right)$ only EUV radiation may heat the gas, while for thicker atmosphere the major role is played by X-rays, since low energy photons have been removed by the upper lying layers of the atmosphere. The results in Fig. 4 do not depend on the electronic fraction (while $Y_{Q}$ does), because our radiative transfer description relies only on the atmospheric abundances of the absorbing species. When column densities are higher than $N_{\mathrm{H}}^{\mathrm{s}}$ any radiation-induced process is suppressed.. Although present in low concentration, heavy elements dominate the gas opacity for primary photo-electrons with energies higher than $0.5 \mathrm{keV}$, and this is reflected in a larger (smaller) column density cutoff for metal rich (poor) atmospheres. For all assumed chemical compositions, beyond approximately $10 \mathrm{keV}$ the main opacity channel is Compton ionization (see Fig. 1), since, as already stated, the total photo-electric cross-section declines sharply with energy.

\section{Discussion and conclusions}

In this work we investigate the role of the EUV and X-ray radiation of a parent star in heating the atmosphere of a giant exoplanet. In order to understand the role of the different spectral energy bands we computed the heating rate and the heating yield assuming a monochromatic stellar photon flux and an atmosphere with the hydrogen component in atomic form, various degrees of fractional ionization $\left(x_{\mathrm{e}}=0.1,10^{-3}, 10^{-6}\right)$ and metallicity $(0.1,1$ and 3 times the standard solar value).
Our analysis shows that for all parameter sets EUV radiation is the dominant source of heating in the upper part of the atmosphere, but it becomes totally ineffective when the hydrogen column density is higher than $N_{\mathrm{H}} \gtrsim 1-10 \times 10^{19} \mathrm{~cm}^{-2}$, which is approximately the column density cut-off for $100 \mathrm{eV}$ photons.

The presence of an energetic cross-over implies that X-rays played an important role in the heating of the hydrogen-rich Earth proto-atmosphere. Smith et al. (2004) estimate for a terrestrial exoplanet atmosphere a column density of the order of $\sim 30-100 \mathrm{~g} \mathrm{~cm}^{-2}$. In such a scenario, results in Fig. 4 suggest that even the hardest X-rays hardly reach the planet surface, regardless of composition of its atmosphere, a conclusion in agreement with Smith et al. (2004). For thinner atmospheres, and for hydrogen escape occurring at a much slower rate than previously thought (Tian et al. 2005b), the solar hard component might have been playing a significant role in terrestrial prebiotic chemistry. However, there are considerable uncertainties in the primordial chemical composition of the anoxic Earth atmosphere, which might contain substantial amount of $\mathrm{CO}_{2}$ (Walker 1985), that is a strong absorber of X-rays (cf. Fig. 4).

We discuss our results in the case of the atmosphere of HD 209458b, that is expected (and largely assumed in models, e.g. García Muñoz 2007) to contain hydrogen, helium and trace amounts of other heavier elements in nearly solar abundances. However, the chemical composition of this exoplanet is far from being understood. Carbon and oxygen have been discovered (Vidal-Madjar et al. 2004), but, e.g., helium remain undetected. For close-in exoplanets beyond the cross-over between stability and instability (Koskinen et al. 2007), all relevant quantities, such as thermal escape rate and the density profile, depend on the heating rate. Nevertheless, by assuming a volume density profile, the column density cut-offs shown in Fig. 4 can be converted to altitudes inside the atmosphere. We adopt the standard hydrostatic equilibrium profile

$n(R)=n_{0} \exp \left[\frac{H}{R_{\mathrm{P}}}\left(1-\frac{R_{\mathrm{P}}}{R}\right)\right]$

where $H$ is the scale height, $R_{\mathrm{P}}$ is the planet radius, and $n_{0}$ is the density at the 1 bar level. In a recent investigation of the physical and chemical aeronomy of HD 209458b, García Muñoz (2007) derived that hydrogen is mostly ionized beyond $R_{\mathrm{f}} \sim 2-4 \times R_{\mathrm{P}}$ with $R_{\mathrm{P}} \sim 1.35 R_{\mathrm{J}}, R_{\mathrm{J}}$ being the Jupiter radius. At that altitude the volume density is not well constrained, and we assume a range of possible values $n\left(R_{\mathrm{f}}\right) \sim 10^{4}-10^{7} \mathrm{~cm}^{-3}$. Assuming $n_{0} \sim 10^{14}-10^{15} \mathrm{~cm}^{-3}$ (Yelle 2004), we obtain by integration of Eq. (6) the column density variation with altitude for the hydrostatic equilibrium profile. The total hydrogen column density up to the 1 bar level is in the range $N_{\mathrm{H}}=2.1-41 \times 10^{22} \mathrm{~cm}^{-2}$. With the same constraints, the EUV column density cut-off $N_{\mathrm{H}}^{\mathrm{s}}=10^{20} \mathrm{~cm}^{-2}$ (upper limit) implies that EUV photons deposit their energy above $R \gtrsim 1.2-1.6 \times R_{\mathrm{p}}$, while $\mathrm{X}$-rays heat the layers below this height. Exploiting a different model density profile (e.g., Yelle 2004 reference model), with $R_{\mathrm{f}} \sim 1.6 \times R_{\mathrm{P}}$, $n_{0} \sim 2 \times 10^{15} \mathrm{~cm}^{-3}$, and $n\left(R_{\mathrm{f}}\right) \sim 2 \times 10^{7} \mathrm{~cm}^{-3}$, appropriate for a hot Jupiter, we obtain a total column density $N_{\mathrm{H}} \sim 6 \times 10^{22} \mathrm{~cm}^{-2}$ and a EUV shielding altitude $R \sim 1.05 \times R_{\mathrm{p}}$. We note that for an equilibrium model characterized by the same set of parameters as in the Yelle (2004) profile, we derive a total column density $N_{\mathrm{H}} \sim 4 \times 10^{23} \mathrm{~cm}^{-2}$, and a EUV shielding altitude of $R \sim 1.2 \times R_{\mathrm{p}}$. Not surprisingly, equilibrium density profiles have EUV shielding altitudes located more externally than model profiles in which the gas density distribution is subject to hydrodynamical escape. Volume and column densities as functions of the altitude for the cases discussed above are shown in Fig. 5. 

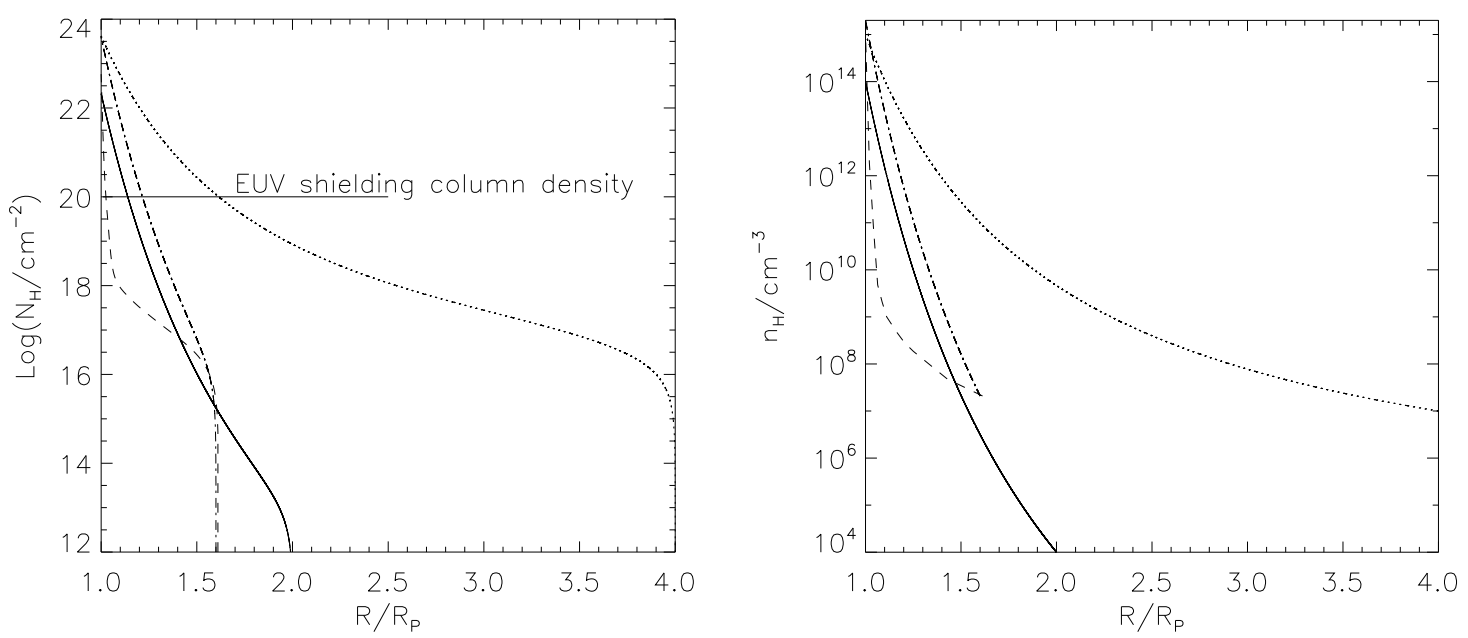

Fig. 5. Column density variation with the altitude inside atmospheres (left panel) whose density profiles (right panel) are described by Eq. (6). The solid line represents the model atmosphere with minimum total column density $\left(R_{\mathrm{f}}=2 \times R_{\mathrm{P}}, n_{0}=1 \times 10^{14} \mathrm{~cm}^{-3}, n\left(R_{\mathrm{f}}\right)=1 \times 10^{4} \mathrm{~cm}^{-3}\right)$ consistent with the assumed range of parameters (see text), while the dotted line represents the atmosphere with maximum total column density $\left(R_{\mathrm{f}}=4 \times R_{\mathrm{P}}, n_{0}=1 \times 10^{15} \mathrm{~cm}^{-3}, n\left(R_{\mathrm{f}}\right)=1 \times 10^{7} \mathrm{~cm}^{-3}\right)$. The case of the Yelle (2004) reference model for HD 209458b is shown for comparison as a dashed line; the dot-dashed curve is the equilibrium model obtained using the parameter set of values derived by Yelle (2004), i.e. $R_{\mathrm{f}}=$ $1.6 \times R_{\mathrm{P}}, n_{0}=2 \times 10^{15} \mathrm{~cm}^{-3}, n\left(R_{\mathrm{f}}\right)=2 \times 10^{7} \mathrm{~cm}^{-3}$.
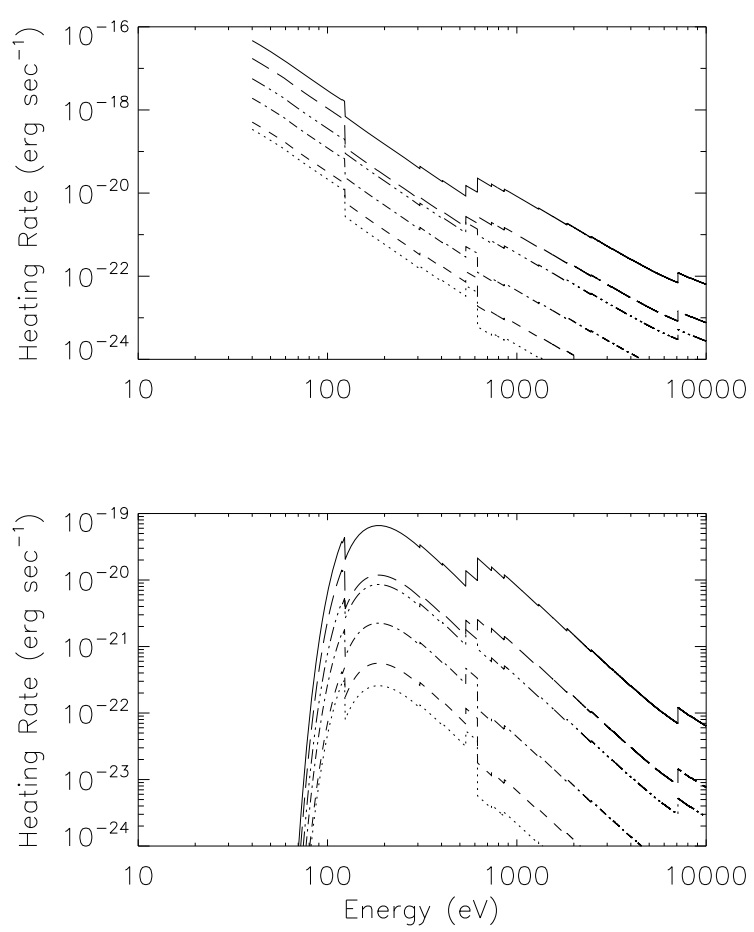

Fig. 6. Heating rate $\left(\mathrm{erg} \mathrm{s}^{-1}\right)$ induced by six stars representative of the evolution of a solar-type star at $1 \mathrm{AU}$ (Ribas et al. 2005). Top panel $N_{\mathrm{H}}=10^{16} \mathrm{~cm}^{-2}$, bottom panel $N_{\mathrm{H}}=10^{20} \mathrm{~cm}^{-2}$. The gas has a standard solar metallicity, the hydrogen is in atomic form and the electron fraction is $x_{\mathrm{e}}=10^{-3}$. From top to bottom the curves are for stellar ages of 0.1, 0.3, 0.65, 1.6, 4.6 and 6.7 Gyr (cf. Ribas et al. 2005).

Since density profiles depend critically on the heating rate, it is important to incorporate an altitude-dependent heating rate coupled with an accurate description of the heating function, in order to derive in a self-consistent way the atmospheric structure. In particular, as atmospheric chemistry is sensitive to the energy of incident radiation, chemical concentrations might depend significantly on the location of the EUV shielding altitude.
Our results can also have implications in the context of the exoplanet's parent star evolution. Current observational evidence indicate that young solar-type stars rotate much faster than the current Sun and that the rotation rate decreases during their evolution on the main sequence, with the result that the XUV emission varies with time, becoming weaker and softer as the star ages (Pizzolato et al. 2003). To estimate the response of our model to XUV emission from solar-type stars of different ages, we used the Ribas et al. (2005) XUV fluxes of six stars, representative of the Sun's main-sequence lifetime from 0.1 to 6.7 Gyr. We convolve $Q_{\text {Xuv }}$ from Eq. (3) with the Ribas et al. (2005) integrated flux (cf. Ribas et al. 2005 Table 4). Figure 6 shows the heating rates as functions of the energy for the six stellar spectra at two column densities, $N_{\mathrm{H}}=10^{16} \mathrm{~cm}^{-2}$ (top panel) and $N_{\mathrm{H}}=10^{20} \mathrm{~cm}^{-2}$ (bottom panel). As in the case of monochromatic unit flux, deep into the atmosphere the heating rate is dominated by the residual hardest component of the spectra. This effect is important at all times during the life of the parent star.

Acknowledgements. This study was carried out within the framework of the ISSI team "Evolution of exoplanet atmospheres and their characterization". This work was supported by the contract ASI-INAF I/088/06/0 and Marie Curie fellowship contract MTKD-CT-2004-002769.

\section{References}

Anders, E., \& Grevesse, N. 1989, Geochim. Cosmochim. Acta, 53, 197 Ballester, G. E., King, D. K., \& Herbert, F. 2007, Nature, 445, 511 Baraffe, I., Selsis, F., Chabrier, G., et al. 2004, A\&A, 419, L13 Baraffe, I., Chabrier, G., Barman, T. S., et al. 2005, A\&A, 436, L47 Ben-Jaffel, L. 2007, ApJ, 671, L61

Berdyugina, S. V., Berdyugin, A. V., Fluri, D. M., \& Piirola, V. 2008, ApJ, 673, L83

Cecchi-Pestellini, C., Ciaravella, A., \& Micela, G. 2006, A\&A, 458, L13

Dalgarno, A., Yan, M., \& Liu, W. 1999, ApJS, 125, 237

Ehrenreich, D., Lecavalier des Etangs, A., Hébrard, G., et al. 2008, A\&A, 483, 933

Favata, F., Flaccomio, E., Reale, F., et al. 2005, ApJS, 160, 469

Feigelson, E. D., Gaffney, J. A., Garmire, G., Hillenbrand, L. A., \& Townsley, L. 2003, ApJ, 584, 911 
García Muñoz, A. 2007, Planet Space Sci., 55, 1246

Guinan, E. F., \& Ribas, I. 2002, in The Evolving Sun and Its Influence on Planetary Environments, ed. B. Montesinos, A. Gimenz, \& E. F. Guinan (San Francisco: ASP), ASP Conf. Ser., 269, 85

Hubbard, W. B., Hattori, M. F., Burrows, A., Hubeny, I., \& Sudarsky, D. 2007, Icarus, 187, 358

Holmström, M., Ekenbäck, A., Selsis, F., et al. 2008, Nature, 451, 970

Koskinen, T. T., Aylword, A. D., \& Miller, S. 2007, Nature, 450, 845

Lammer, H., Selsis, F., Ribas, I., et al. 2003, ApJ, 598, L121

Micela, G. 2002, in The evolving Sun and Its Influence on Planetary Environments, ed. B. Montesinos, A. Gimenez, \& E. F. Guinan (San Francisco: ASP), ASP Conf. Ser., 269, 107

Osterbrock, D. E. 1989, Astrophysics of Gaseous Nebulae and Active Galctic Nuclei (Sausalito: University Science Books)

Penz, T., \& Micela, G. 2008, A\&A, 479, 579

Penz, T., Micela, G., \& Lammer, H. 2008, A\&A, 477, 309

Pizzolato, N., Maggio, A., Micela, G., Sciortino, S., \& Ventura, P. 2003, A\&A, 397,147
Ribas, I., Guinan, E. F., Gudel, M., \& Audard, M. 2005, ApJ, 622, 680

Sobel'man, I. 1991, Atomic Spectra and Radiative Transitions (Berlin: Springer) Smith, D. S., Scalo, J., \& Wheeler, J. C. 2004, Icarus, 171, 229.

Tian, F., Toon, O. B., Pavlov, A., \& De Sterck, H. 2005, ApJ, 621, 1049

Tian, F., Toon, B. O., Pavlov, A. A., \& De Sterck, H. 2005, Science, 308, 1014

Verner, D. A., Yakovlev, D. G., Band, I. M., \& Trzhaskovskaya, M. B. 1993, Atomic Data and Nuclear Data Tables, 55, 233

Vidal-Madjar, A., Lecavalier des Etangs, A., Désert, J.-M., et al., 2003, Nature, 422, 143

Vidal-Madjar, A., Désert, J.-M., Lecavelier des Etangs, A., et al. 2004, ApJ, 604, L69

Vidal-Madjar, A., Lecavalier des Etangs, A., Désert, J.-M., et al. 2008, ApJ, 676, L57

Walker, J. C. G. 1985, Orig. Life, 16, 117

Yan, M., Sadeghpour, H. R., \& Dalgarno, A. 1998, ApJ, 496, 1044

Yelle, R. V. 2004, Icarus, 170, 167

Yelle, R. V. 2006, Icarus, 183, 508 